\title{
Hybrid single quantum well InP/Si nanobeam lasers for silicon photonics
}

\author{
William S. Fegadolli, ${ }^{1,2 * *}$ Se-Heon Kim, ${ }^{2,3}$ Pablo Aitor Postigo, ${ }^{4}$ and Axel Scherer ${ }^{1,2}$ \\ ${ }^{1}$ Department of Electrical Engineering, California Institute of Technology, Pasadena, California 91125, USA \\ ${ }^{2}$ Department of Physics, California Institute of Technology, Pasadena, California 91125, USA \\ ${ }^{3}$ Currently with Hitachi Global Storage Technologies, San Jose, California 95138, USA

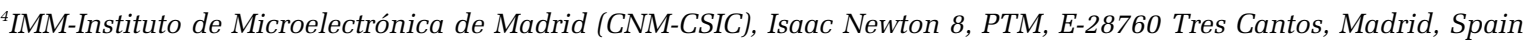 \\ *Corresponding author: fegadoli@caltech.edu
}

Received August 27, 2013; revised October 7, 2013; accepted October 7, 2013; posted October 8, 2013 (Doc. ID 196423); published November 8, 2013

\begin{abstract}
We report on a hybrid InP/Si photonic crystal nanobeam laser emitting at $1578 \mathrm{~nm}$ with a low threshold power of $\sim 14.7 \mu \mathrm{W}$. Laser gain is provided from a single InAsP quantum well embedded in a $155 \mathrm{~nm}$ InP layer bonded on a standard silicon-on-insulator wafer. This miniaturized nanolaser, with an extremely small modal volume of $0.375(\lambda / n)^{3}$, is a promising and efficient light source for silicon photonics. (c) 2013 Optical Society of America OCIS codes: (130.0130) Integrated optics; (140.0140) Lasers and laser optics; (160.5298) Photonic crystals. http://dx.doi.org/10.1364/OL.38.004656
\end{abstract}

Silicon photonics has been considered a promising technology, owing to its intrinsic characteristic of allowing high integration of optical devices in small footprints and to its synergy with existing complementary metaloxide semiconductor (CMOS) processes. The applications have promised to cover a wide spectrum, comprising conventional long-distance down to intrachip communications, optical sensors, and spectroscopy systems in general $[\underline{1}, 2]$.

For the past decade, several research groups have demonstrated essential building blocks to process optical signals, such as efficient and broadband input/output coupling systems from optical fibers to optical waveguides []ㅡ], high-speed electro-optic modulators [ㄴ] ], filters []], tunable filters []], reconfigurable switches [7]], unidirectional devices [8], and others.

However, the capability of generating light on silicon has been one of the daunting problems to overcome owing to its indirect electronic bandgap [9]. Faced with this long-standing issue, several research groups have proposed heterogeneous integration of III-V quantum well (QW) materials on top of silicon structures to generate light at the telecommunication wavelengths [9-15].

In this Letter, we demonstrate a novel low threshold and optically pumped single QW nanobeam laser, defined by means of a heterogeneous integration of an InP layer (with a single $\operatorname{InAs}_{0.65} \mathrm{P}_{0.35} \mathrm{QW}$ embedded) on silicon-on-insulator.

First, we focused on the design of the heterogeneous $\mathrm{InP} / \mathrm{Si}$ nanobeam cavity and pursued a high $Q$-factor in an attempt to reduce the lasing threshold. Simulations were made using finite-difference time-domain (FDTD) models, where the well-known techniques to design 1D photonics crystal nanobeam cavities with the desired figures of merits were employed [16-19].

Preliminarily simulations showed that a hybrid bilayer of $155 \mathrm{~nm}$ thick $\mathrm{InP}$ on top of $220 \mathrm{~nm}$ thick silicon-oninsulator, $500 \mathrm{~nm}$ wide, and holes suitably designed can form a heterogeneous nanobeam cavity with a theoretical $Q$-factor as high as $2.27 \times 10^{6}$ and modal volume as low as $0.375(\lambda / n)^{3}$. To attain such figures of merit, the reflector sections of the cavity are designed with a periodic structure of 13 holes, drilled through both layers of InP and $\mathrm{Si}$, with periodicity $\alpha=370 \mathrm{~nm}$. In addition, a linear tapered section (from $0.28 \alpha$ to $0.22 \alpha$ ) is suitably designed with five holes, drilled through both layers, to precisely phase match the waveguide and Bloch modes [17]. Finally, the $Q$-factor and resonant wavelength are tailored by varying the length of the dielectric section on the center of the nanobeam cavity $[S$ in Fig. 1(b)].

Figure 1(a) shows the theoretical $Q$-factor and resonant wavelength of the heterogeneous $\mathrm{Si} / \mathrm{InP}$ nanobeam

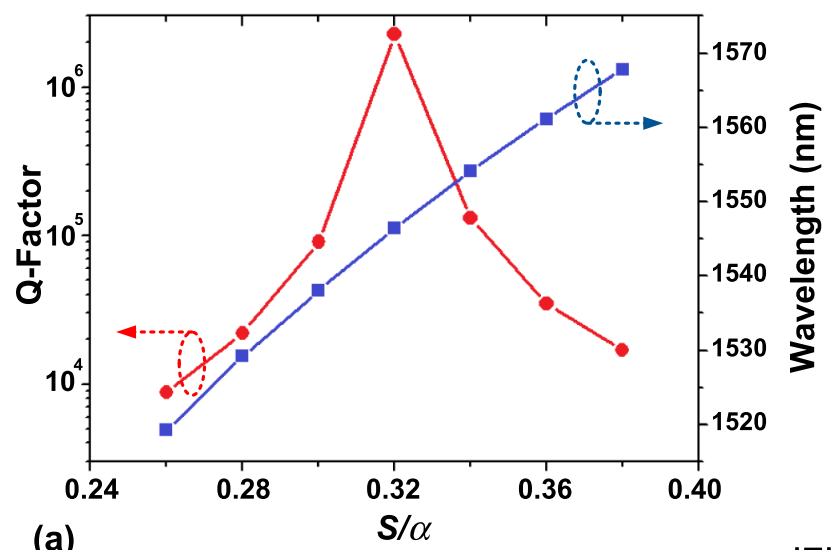

(a)
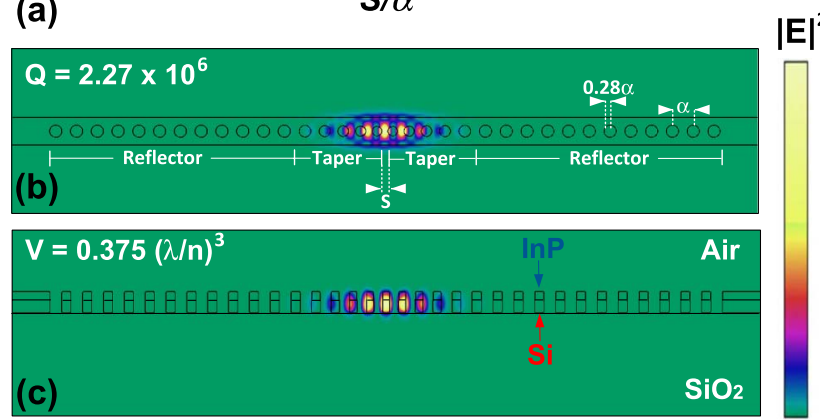

Fig. 1. (a) Theoretical $Q$-factor and resonant wavelength as a function of the ratio between $S$ and $\alpha$. (b) and (c) are, respectively, top and longitudinal view for the theoretical optical resonant mode. 
cavity as a function of ratio between $S$ and $\alpha$. Figures 1(b) and $1(\mathrm{c})$ show, respectively, the theoretical resonant optical mode for the highest theoretical $Q$-factor for the top and longitudinal view as well as the schematic representation of the device.

It would be still challenging to achieve a lasing threshold because the laser gain is provided from only a single, $5 \mathrm{~nm}$ thick, QW layer. Moreover, any minute imperfection introduced during the fabrication processes could severely lower the $Q$ as Fig. 1(a) implies.

Therefore, we evaluated the required threshold gain coefficient using the formulation proposed by Chang and Chuang [20]:

$$
g_{\mathrm{th}}=\frac{1}{\Gamma_{E} \cdot Q} \frac{2 \pi n_{g, a}}{\lambda},
$$

where $n_{g, a}$ is the material group index of the QW, and $\Gamma_{E}$ is the energy confinement factor [20]. With $\Gamma_{E}$ of $0.7 \%$ (obtained by FDTD) and the theoretical $Q$ of $2.27 \times 10^{6}$, we estimated $g_{\text {th }}$ to be $7.5 \mathrm{~cm}^{-1}$ on the ideal case. Note that $g_{\text {th }} \sim 1000 \mathrm{~cm}^{-1}$ is easily achievable from InAsP/InP material systems. Therefore, even with a 100 times degraded $Q\left(2.27 \times 10^{4}\right)$, lasing is possible to achieve.

The device was fabricated by means of standard nanofabrication processes compatible with today's CMOS fabrication procedures.

First, we prebonded a stack of III-V wafer (155 nm thick InP with a single $\operatorname{InAs}_{0.65} \mathrm{P}_{0.35}$ QW embedded in the middle/100 nm thick InGaAs etch stop layer/InP substrate) on top of a SOI wafer ( $220 \mathrm{~nm}$ thick $\mathrm{Si} / 2 \mu \mathrm{m}$ thick $\mathrm{SiO}_{2} / a$ Si substrate) by means of assisted oxygen plasma activation [21]. Then we applied a pressure of $0.1 \mathrm{MPa}$ at $300^{\circ} \mathrm{C}$ for $2 \mathrm{~h}$ to obtain strong covalent bonding. After completion of bonding, the InP substrate was etched away in diluted $\mathrm{HCl}(\mathrm{HCl}$ : Water $=4: 1)$ at $4^{\circ} \mathrm{C}$, which took approximately $3 \mathrm{~h}$. Finally, the InGaAs etch stop layer was removed by means of a compounded etch solution of $\mathrm{HF}, \mathrm{Cr}_{2} \mathrm{O}_{3}$, and DI water, resulting in a thin layer of InP/InAsP membrane bonded on top of $220 \mathrm{~nm}$ thick $\mathrm{Si}$. A single step of electron-beam lithography, using negative-tone resist (hydrogen silsesquioxane), was performed to define a set of nanobeam cavities with varying lattice constants. The pattern was transferred by means of two-step dry etching. First, the InP layer was etched by a chemically assisted ion-beam etching (CAIBE) with $\mathrm{Cl}_{2}$ and followed by reactive ion etching using a mixture of $\mathrm{SF}_{6}$ and $\mathrm{C}_{4} \mathrm{~F}_{8}$.

Figure 2 shows SEM images of the fabricated device under different magnifications and perspectives. One can observe well-defined holes and a uniformly bonded InP layer on top of Si. The small undercut in the InP layer is visible nearby the bonding interface $(\sim 15 \mathrm{~nm})$, while the silicon sidewalls are vertical and smooth.

Lasing characteristics of the devices were analyzed using pumping and detection of the emission normal to the sample. A laser diode with a wavelength of $830 \mathrm{~nm}$ was used to pump the laser. Figure 3(a) shows a typical lasing spectrum and a lasing-mode pattern recorded by a charge-coupled device camera (inset). In fact, a significant amount of the pump energy

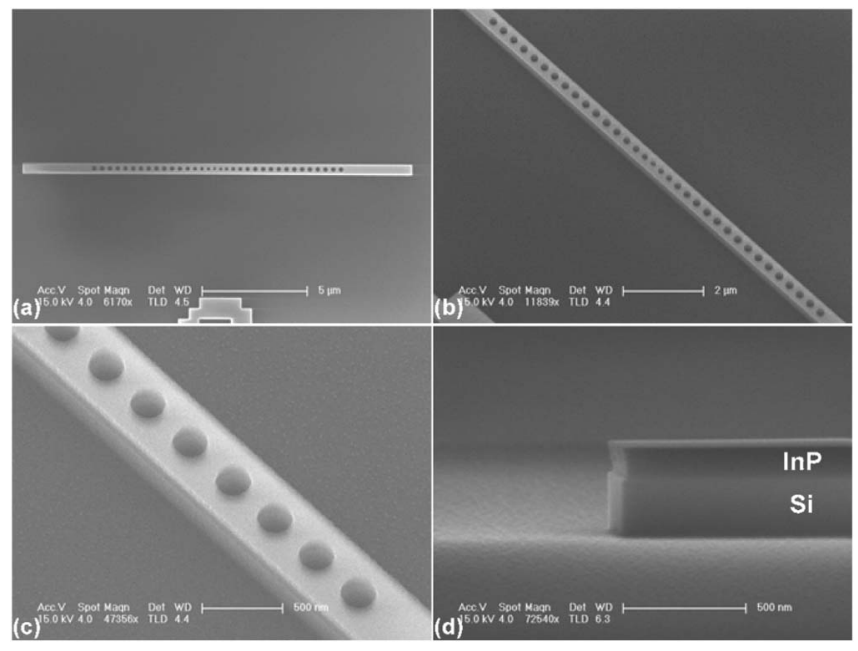

Fig. 2. (a)-(d) are the device's micrographs under different angles and magnifications obtained by means of scanning electronic microscope (SEM).

(830 nm) also can be absorbed by the underlying Si layer, which causes heating. It is worthy to point out that this thermal issue can be relieved by employing a new III-V material system of InGaAsP QWs that can be efficiently excited by a longer wavelength pump laser at which $\mathrm{Si}$ is almost transparent. The device was driven in a pulsed mode; a pulse period of $1 \mu \mathrm{s}$ and duty cycle of $1.5 \%-3 \%$.

In Fig. 3(b), we plot a light-in versus light-out $(L-L)$ curve for the device shown in Fig. 3(a) under the pulse pumping (duty 1.5\%). Here, using a rigorous FDTD

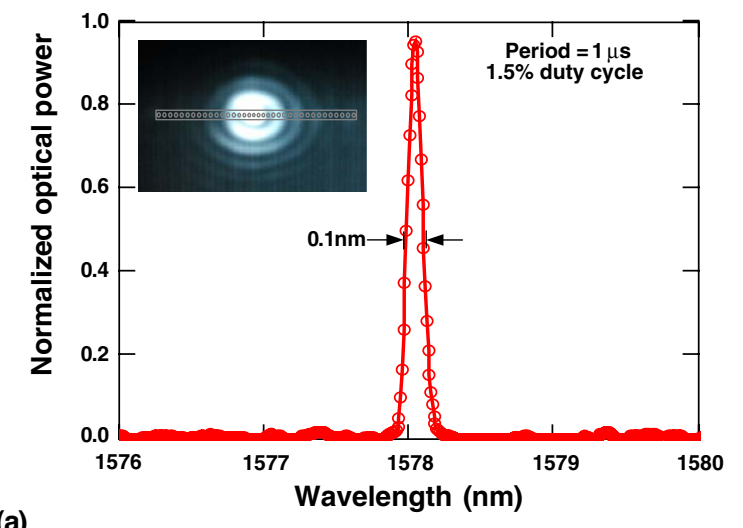

(a)

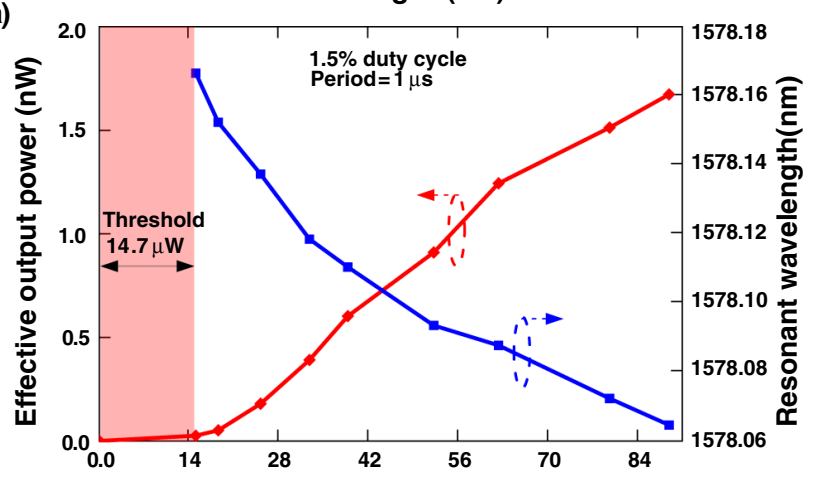

(b)

Effective peak pump power $(\mu \mathrm{W})$

Fig. 3. (a) Lasing spectrum and the corresponding laser mode pattern as an inset. (b) Output power (red) and resonant wavelength (blue) as a function of input power. 


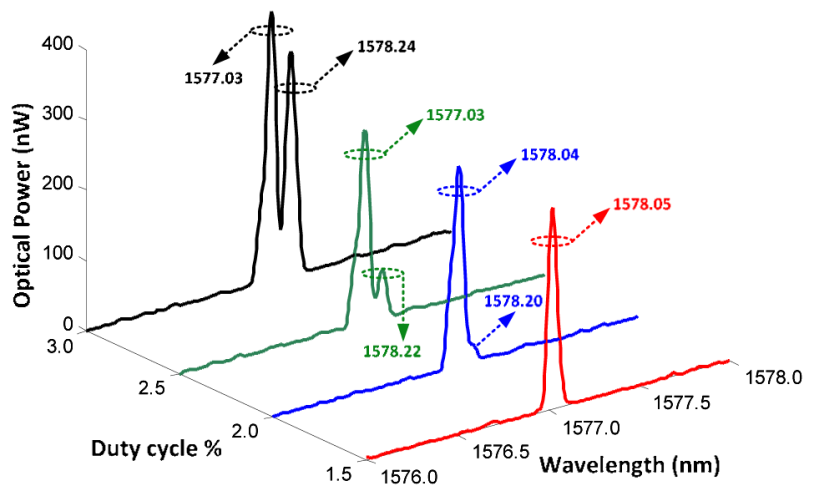

Fig. 4. Laser's response as a function of wavelength for different duty cycles.

simulation [22], we estimate that about $7 \%$ of the actual irradiated power can be absorbed in the InP slab, which accounts for the following two factors: (1) the areal mismatch between the pump beam and the nanobeam and (2) multiple reflections in the high index slab [23]. We believe that a quite low peak threshold value of $\sim 14.7 \mu \mathrm{W}$ measured from this plot is probably attributed to the nanobeam cavity's large $\mathrm{Q} / \mathrm{V}$.

Interestingly, in the above threshold regime, a peak lasing wavelength blueshifts as we increase the pump power within the range demonstrated by Fig. 3(b). Though the absolute change in wavelength was small $(100 \mathrm{pm})$, this behavior implies that the carrier plasma effect is still present along with the well-known thermo-optic effect. This result also shows that the free carriers in the underlying Si layer cannot be depleted through the stimulated emission process (thus its density ever increases with the pump power even in the above threshold).

On the other hand, as we increase the duty cycle, the thermo-optic effect can be seen along with the aforementioned carrier-plasma effect. We observed that the output power for thermal rollover takes place when the input power is around 10 times the threshold power.

In Fig. 4 , one can observe that a new peak grows on the longer wavelength side as the duty cycle is increased. Here, it should be emphasized that such effect does not mean coupling of energy into two orthogonal resonant modes because the nanobeam cavity's mode volume is too small to support such closely located two modes. Rather, the observed peak splitting indicates that the peak wavelength is changing in time. Note that the optical spectrum analyzer used in this experiment (Ando AQ6317) has a rather slow integration time of 1-10 ms.

In summary, we have experimentally demonstrated the single QW InP/Si nanobeam laser emitting at a telecommunication wavelength. This small and power-efficient proof-of-concept laser device finds important applications in silicon photonics, as a light source and an optical modulator, brings up the integration density at least by a factor of $10-100$.
W. S. F., S. K., and A. S. thank the NSF CIAN ERC (Grant EEC-0812072) and Sanofi S. A. for the financial support, as well as P. A. P. thank the Spanish MICINN (Grant FA8655-12-1-2125) for financial support.

\section{References}

1. L. Pavesi and G. Guillot, Optical Interconnects-The Silicon Approach (Springer-Verlag, 2006).

2. S. M. Grist, S. A. Schmidt, J. Flueckiger, V. Donzella, W. Shi, S. T. Fard, J. T. Kirk, D. M. Ratner, K. C. Cheung, and L. Chrostowski, Opt. Express 21, 7994 (2013).

3. V. R. Almeida, R. R. Panepucci, and M. Lipson, Opt. Lett. 28, 1302 (2003).

4. D. J. Thomson, F. Y. Gardes, Y. Hu, G. Mashanovich, M. Fournier, P. Grosse, J.-M. Fedeli, and G. T. Reed, Opt. Express 19, 11507 (2011).

5. F. Xia, M. Rooks, L. Sekaric, and Y. Vlasov, Opt. Express 15, 11934 (2007).

6. P. Dong, W. Qian, H. Liang, R. Shafiiha, D. Feng, G. Li, J. E. Cunningham, A. V. Krishnamoorthy, and M. Asghari, Opt. Express 18, 20298 (2010).

7. W. S. Fegadolli, G. Vargas, X. Wang, F. Valini, L. A. M. Barea, J. E. B. Oliveira, N. Frateschi, A. Scherer, V. R. Almeida, and R. R. Panepucci, Opt. Express 20, 14722 (2012).

8. L. Feng, Y. Xu, W. S. Fegadolli, M. Lu, J. E. B. Oliveira, V. R. Almeida, Y. Chen, and A. Scherer, Nat. Mater. 12 108 (2012).

9. D. Liang and J. E. Bowers, Nat. Photonics, 4, 511 (2010).

10. J. Van Campenhout, P. R. Romeo, P. Regreny, C. Seassal, D. Van Thourhout, S. Verstuyft, L. Di Cioccio, J.-M. Fedeli, C. Lagahe, and R. Baets, Opt. Express 15, 6744 (2007).

11. D. Liang, M. Fiorentino, T. Okumura, H. Chang, D. T. Spencer, Y. Kuo, A. W. Fang, D. Dai, R. G. Beausoleil, and J. E. Bowers, Opt. Express 17, 20355 (2009).

12. H. Park, A. Fang, S. Kodama, and J. Bowers, Opt. Express 13, 9460 (2005).

13. G. Roelkens, D. Van Thourhout, R. Baets, R. Nötzel, and M. Smit, Opt. Express 14, 8154 (2006).

14. X. Sun, A. Zadok, M. J. Shearn, K. A. Diest, A. Ghaffari, H. A. Atwater, A. Scherer, and A. Yariv, Opt. Lett. 34, 1345 (2009).

15. B. B. Bakir, A. Descos, N. Olivier, D. Bordel, P. Grosse, E. Augendre, L. Fulbert, and J. M. Fedeli, Opt. Express 19, 10317 (2011).

16. P. B. Deotare, M. W. McCutcheon, I. W. Frank, M. Khan, and M. Loncar, Appl. Phys. Lett. 94, 121106 (2009).

17. C. Sauvan, G. Lecamp, P. Lalanne, and J. P. Hugonin, Opt. Express 13, 245 (2005).

18. P. Lalanne and J. P. Hugonin, IEEE J. Quantum Electron. 39, 1430 (2003).

19. W. S. Fegadolli, J. E. B. Oliveira, V. R. Almeida, and A. Scherer, Opt. Express 21, 3861 (2013).

20. S.-W. Chang and S. L. Chuang, IEEE J. Quantum Electron. 45, 1014 (2009).

21. D. Pasquariello and K. Hjort, IEEE J. Sel. Top. Quantum Electron. 8, 118 (2002).

22. S. Kim, R. W. Day, J. F. Cahoon, T. J. Kempa, K. Song, H. Park, and C. M. Lieber, Nano Lett. 12, 4971 (2012).

23. S. Kita, K. Nozaki, S. Hachuda, H. Watanabe, Y. Saito, S. Otsuka, T. Nakada, Y. Arita, and T. Baba, IEEE J. Sel. Top. Quantum Electron. 17, 1632 (2011). 